Published in final edited form as:

Anal Chem. 2019 March 19; 91(6): 4010-4016. doi:10.1021/acs.analchem.8b05399.

\title{
Characterization and Optimization of Multiplexed Quantitative Analyses Using High-Field Asymmetric-Waveform Ion Mobility Mass Spectrometry
}

\author{
Devin K. Schweppe ${ }^{\star}, \dagger$, Satendra Prasad ${ }^{\ddagger}$, Michael W. Belford ${ }^{\ddagger}$, José Navarrete-Perea ${ }^{\dagger}$, \\ Derek J. Bailey ${ }^{\ddagger}$, Romain Huguet ${ }^{\ddagger}$, Mark P. Jedrychowski ${ }^{\dagger}$, Ramin $\operatorname{Rad}^{\dagger}$, Graeme \\ McAlister $\ddagger$, Susan E. Abbatiello $\ddagger$, Eloy R. Woulters ${ }^{\ddagger}$, Vlad Zabrouskov ${ }^{\ddagger}$, Jean-Jacques \\ Dunyach $^{\ddagger}$, João A. Paulo ${ }^{\dagger}$, Steven P. Gygi ${ }^{\star}, \dagger$ \\ †Department of Cell Biology, Harvard Medical School, Cambridge, Massachusetts 02115, United \\ States \\ ¥Thermo Scientific LSMS, San Jose, California 95134, United States
}

\begin{abstract}
Multiplexed, isobaric tagging methods are powerful techniques to increase throughput, precision, and accuracy in quantitative proteomics. The dynamic range and accuracy of quantitation, however, can be limited by coisolation of tag-containing peptides that release reporter ions and conflate quantitative measurements across precursors. Methods to alleviate these effects often lead to the loss of protein and peptide identifications through online or offline filtering of interference containing spectra. To alleviate this effect, high-Field Asymmetric-waveform Ion Mobility Spectroscopy (FAIMS) has been proposed as a method to reduce precursor coisolation and improve the accuracy and dynamic range of multiplex quantitation. Here we tested the use of FAIMS to improve quantitative accuracy using previously established TMT-based interference

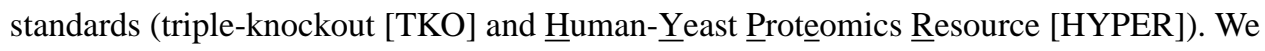
observed that FAIMS robustly improved the quantitative accuracy of both high-resolution $\mathrm{MS}^{2}$ $\left(\mathrm{HRMS}^{2}\right.$ ) and synchronous precursor selection $\mathrm{MS}^{3}$ (SPS-MS ${ }^{3}$ )-based methods without sacrificing protein identifications. We further optimized and characterized the main factors that enable robust use of FAIMS for multiplexed quantitation. We highlight these factors and provide method recommendations to take advantage of FAIMS technology to improve isobaric-tag-quantification moving forward.
\end{abstract}

\section{Graphical Abstract}

\footnotetext{
*Corresponding Authors: devin_schweppe@hms.harvard.edu.; steven_gygi@hms.harvard.edu. Supporting Information

The Supporting Information is available free of charge on the ACS Publications website at DOI: 10.1021/acs.analchem.8b05399. Supplementary methods and additional data, including unlabeled peptide identification rates based on FAIMS CV, comparison of total unique peptides identified using various FAIMS HRMS ${ }^{2}$ methods, maximum observable ratio, and other data (PDF)

Number of CVs used, quantitation method, scan number for acquisition, observed mass to charge for the precursor, precursor state charge, and additional data (XLSX)

The authors declare the following competing financial interest(s): S.P., M.B. D.J.B, R.H., G.M., S.E.A., E.R.W., V.Z., and J.J.D. are employees of ThermoFisher Scientific, the manufacturer of the FAIMS Pro source.
} 


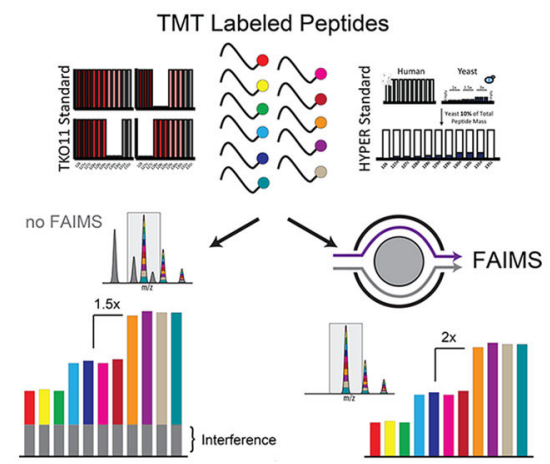

Sample multiplexing for quantitative proteomics enables improved precision, reduced missing values, and increased throughput. The canonical methods to multiplex multiple samples within a single sample injection rely on isobaric tags (e.g., iTRAQ and TMT). ${ }^{1,2}$ The flexibility and utility of isobaric tagging methods has been extended to a range of biological questions from single-cell proteomics to large clinical cohort studies. ${ }^{2}$ However, multiplexed quantitative methods must contend with ratio compression due to channel interference wherein coisolation of undesired isobaric-tag containing peptide fragments results in attenuation of the quantitative dynamic range and convergence of quantitative ratios toward the median. ${ }^{1-3}$ The standard high-resolution $\mathrm{MS}^{2}\left(\mathrm{HRMS}^{2}\right)$ methods are particularly vulnerable to this issue as they rely on only two precursor filtering mechanisms, chromatography and a single precursor isolation width, to remove interfering ions. To alleviate the effects of interference, several methods had been proposed to reduce or compensate for the effects of this ratio compression. These include improving chromatographic resolution, using gas phase fractionation, adding ion mobility separations, shrinking precursor isolation windows, complement ion quantification and stringent interference filters. $^{4-7}$

One of the most notable improvements in quantitative accuracy was achieved with the advent of methods that applied secondary fragmentation of TMT containing ions followed by $\mathrm{MS}^{3}$ scans for quantitation. ${ }^{3,8}$ Selection of individual fragment ions from an $\mathrm{MS}^{2}$ for further fragmentation and quantitation at the $\mathrm{MS}^{3}$ level reduces the negative effect of coisolating ions in $\mathrm{HRMS}^{2}$ spectra allowing more accurate quantitative ratios. ${ }^{8}$ Quantification at the $\mathrm{MS}^{3}$ level does not require $\mathrm{HRMS}^{2}$ scans, instead making use of ion trap scans for peptide spectral matching and $\mathrm{MS}^{2}$-fragment selection. Furthermore, the development of multinotch $\mathrm{MS}^{3}$, or synchronous precursor selection $\mathrm{MS}^{3}\left(\mathrm{SPS}^{-\mathrm{MS}^{3}}{ }^{3}\right.$ ), methods improved the utility of $\mathrm{MS}^{3}$-based quantitation by increasing the number of ions that could be isolated for secondary fragmentation and thereby increasing the total TMT signal that could be measured in the $\mathrm{MS}^{3}$ scans. ${ }^{3,8}$ Improved accuracy using SPS-MS ${ }^{3}$ methods comes at the price of reduced peptide and protein identifications due to sacrificing run time for the acquisition of high-resolution $\mathrm{MS}^{3}$ spectra. ${ }^{1}$ The increased time component means that fewer peptide-matching scans can be collected across the chromatographic space. Even considering the reduced protein identification rate, the improved quantitative accuracy of SPS-MS ${ }^{3}$ based methods highlights the advantage that can be gained by eliminating the effects of precursor coisolation in multiplexed analyses. ${ }^{3,8}$ 
Recent work has shown the potential advantage of the separation of precursors by their ion mobilities to reduce precursor coisolation, including techniques such as high-Field Asymmetric-waveform Ion Mobility Spectroscopy (FAIMS) sources. ${ }^{4,9}$ Thermo Fisher developed a FAIMS source (the FAIMS Pro) with a cylindrical central electrode that has low resolving power but significantly improved ion transmission. ${ }^{10-12}$ The new FAIMS source can separate precursor ions at atmospheric pressure in the gas phase based on their mobility through a strong electric field $\left(33000 \mathrm{eV} / \mathrm{cm}^{3}\right)$. In a FAIMS device, ions are propelled between two concentric cylinders separated by a gap $(g=1.5 \mathrm{~mm}$ to $2.5 \mathrm{~mm}$ ) using a carrier gas (i.e., nitrogen). In the gap, ions experience a bisinusoidal RF waveform commonly termed as the Dispersion Voltage (DV) with an alternating high $(\mathrm{DV}=-5000 \mathrm{~V})$ and low $(\mathrm{DV}=-1700 \mathrm{~V})$ amplitude at a $3 \mathrm{MHz}$ frequency. The field strength in the gap is reported as Electric Field normalized to Number Gas Density $(\mathrm{E} / \mathrm{N})$ in units of Townsend $\left(\mathrm{Td}=10^{-21}\right.$ $\left.\mathrm{V}^{*} \mathrm{~m}^{2}\right)$ and at high $\mathrm{E} / \mathrm{N}(\sim 186 \mathrm{Td})$ such as that established during the high period of the waveform with heated electrodes $\left(100{ }^{\circ} \mathrm{C}\right)$, ions experience a change in their average collisional cross section relative to the that in the low field. Consequently, ions exert different electrical mobilities during the high $\left(K_{\mathrm{h}}\right)$ and low $\left(K_{1}\right)$ period of the waveform, resulting in a difference in mobility $(\Delta K)$ per RF cycle. During transit $(t=20-50 \mathrm{~ms})$ through the electrodes ions experience millions of RF cycles producing a net difference in mobility that causes ions to deviate toward one of the FAIMS electrodes. A DC offset or Compensation Voltage (CV) can be applied to one of the electrodes to restore ion trajectory toward the center of the gap for transmission, and the FAIMS source can cycle through multiple CVs within a single LC-MS/MS method. Because ions are separated based on $\Delta K$, the FAIMS technique can be seen as partially orthogonal to $m / z$ and can be expected to distinguish between precursor ions even when they have similar observed $\mathrm{m} / z$. This technique has the added advantage of increased speed as it operates in gaseous phase as opposed to condensed phase thus providing the orthogonal separation dimension with little to no time cost. Hebert et al. and Pfammatter et al. leveraged the FAIMS technique as a gasphase fractionation tool analogous to offline condensed phase fractionation. ${ }^{10,13}$ In these studies, FAIMS provided similar depth, for tryptic peptides derived from K562 or HEK293 cells, compared to the analysis of LC fractions, over a fixed analysis time.

In this work, we characterized and optimized the utility of FAIMS for the efficient separation of coeluting precursor ions in multiplexed proteomics analyses. We further measured the effect of reducing this precursor coelution had on reporter-ion interference in multiplexed quantitative analyses on a Thermo Orbitrap Fusion Lumos (Figure 1A). We observed up to a 6-fold reduction in quantitative interference using FAIMS without sacrificing protein identification rates. Using a TMT-interference standard ${ }^{14}$ we determined that FAIMS significantly improved the quantitative accuracy of multiplexed analyses for both HRMS $^{2}$ and SPS-MS 3 methods. Furthermore, we highlight the factors that contribute to improving the quantitative accuracy of both $\mathrm{HRMS}^{2}$ and SPS-MS ${ }^{3}$ methods and provide recommendations for future integration of the FAIMS technology into multiplexed proteomic strategies. 


\section{EXPERIMENTAL SECTION}

\section{Sample Preparation.}

Yeast cells (Saccharomyces cerevisae, BY4742) were grown in $500 \mathrm{~mL}$ of YPD cultures to $\mathrm{OD}_{600}=0.8$. Yeast cells were washed twice with ice-cold PBS, pelleted, and stored at $-80 \mathrm{C}$ until use. Yeast cells were lysed by resuspending in lysis buffer ( $8 \mathrm{M}$ urea, $50 \mathrm{mM}$ EPPS pH $8.5,150 \mathrm{mM} \mathrm{NaCl}$, Roche protease inhibitor tablet) and bead beating (Supplementary Methods). After lysis and bead removal, the lysate was centrifuged to remove cellular debris, and the supernatant was collected for use. Human HCT116 cell pellets were lysed, and lysates were reduced and alkylated prior to digestion with LysC/Trypsin (Supplementary Methods). Peptide digests were aliquoted to desired concentrations and labeled with separate TMT channels. The labeled peptides were mixed and desalted (50 mg $\mathrm{C}_{18}$ SepPak, Waters) prior to use.

\section{Mass Spectrometric Data Acquisition and Data Analysis.}

Labeled peptides were resuspended in $5 \% \mathrm{ACN} / 2 \%$ formic acid at $1 \mathrm{mg} / \mathrm{mL}$ and loaded at 1 $\mu \mathrm{g}$, unless otherwise noted, on an in-house pulled $\mathrm{C}_{18}$ column $(30 \mathrm{~cm}, 2.6 \mathrm{um}$ Accucore [Thermo Fisher], 100um ID), and eluted using a linear gradient from $0 \%$ to $30 \%$ buffer B (95\% ACN, $0.125 \%$ formic acid). Eluted peptides were injected into an Orbitrap Fusion Lumos (Tune 3.1.2412) using a either a high-resolution $\mathrm{MS}^{2}\left(\mathrm{HRMS}^{2}\right.$ ) or synchronous precursor selection (SPS-MS ${ }^{3}$ ) method for quantitation (Supplementary Methods). All figure panels include replicate injections separated on the same analytical column. Acquired Raw files were searched with SEQUEST ${ }^{15}$ using an in house proteomic pipeline (Supplementary Methods). Peptide spectral matches were first filtered to a peptide false discovery rate of less than $1 \%$ based on linear discriminant analysis using a target decoy strategy. ${ }^{16,17}$ Peptides were subsequently filtered to a final protein-level false discovery rate less than $1 \% .{ }^{16,18}$ For quantitation, a total sum signal-to-noise of all reporter ions of 200 was required for analysis and comparisons. Data analysis was performed using the R statistical scripting language (3.5.1, "Feather Spray"). ${ }^{19}$

\section{High-Field Asymmetric-waveform Ion Mobility Spectroscopy (FAIMS).}

The Thermo FAIMS Pro device was run with default parameters. Briefly, the FAIMS source was operated in standard resolution which sets the FAIMS inner and outer electrode to $100{ }^{\circ} \mathrm{C}$ and yields a FWHM between $10 \mathrm{~V}$ to $15 \mathrm{~V}$ (Supplementary Methods). No additional gas was supplied for desolvation. The DV circuitry was tuned using the autotune option, which independently tunes each of the sine waves and phase shifts one of the waveforms by $\pi / 2$ to assemble a bisinusoidal waveform with a high amplitude of $-5000 \mathrm{~V}$ at a $3 \mathrm{MHz}$ frequency. The CV switch time was automatically calculated based on the capillary conductance and was set to $25 \mathrm{~ms}$ (Orbitrap Fusion Lumos).

\section{Measurement of Quantitative Interference and Dynamic Range.}

The interference free index (IFI) was calculated as previously described. ${ }^{14}$ The maximum observable ratio (MOR) for TKO samples was the proportional measure of the estimated 
maximum ratio that could be measured based on the amount of interference observed in the sample with a given instrument method (Supplementary Methods).

\section{RESULTS AND DISCUSSION}

Reducing the effect of precursor coisolation for isobaric-tag based quantitative proteomics has the potential to vastly improve the dynamic range, accuracy, and precision of multiplexed analyses. Differential ion mobility prior to mass isolation separates precursors based on mass and charge (Figure 1A,B). To accomplish differential ion mobility separation we used a new, high ion-transmission, low-resolving-power FAIMS device (FAIMS Pro, Thermo Fisher Scientific). As an initial assessment of the separation of precursors by differential ion mobility we compared the charge states of $\mathrm{MS}^{1}$ features at CVs from $-20 \mathrm{~V}$ to $-80 \mathrm{~V}$ (Figure 1B). Precursors with +1 charge state were enriched at CVs greater than $-40 \mathrm{~V}$, whereas $+2 /+3 /+4$ charge state precursors were enriched at CVs less than $-40 \mathrm{~V}$. Qualitative comparison of the triple knockout (TKO) standard ${ }^{14}$ or the two proteome Human Yeast Proteomics Resource (HYPER) standard resulted in noticeable, consistent differences in the total ion current chromatograms over multiple individual CVs (Figure 1C,D). Thus, the precursor separation, and its potential benefits, were consistent for both a low (TKO) and high (HYPER) complexity samples. Together these observations lent credence to our supposition that FAIMS could be useful for mobility-based prefractionation of precursor ions prior to mass analysis.

Optimal CVs for peptide and protein identifications were determined by replicate injections of the TKO standard at decreasing CVs from $-20 \mathrm{~V}$ to $-100 \mathrm{~V}$ (one CV per sample injection). A CV of $-50 \mathrm{~V}$ generated the highest number of TMT-labeled peptide identifications (Figure 2A). CVs between $-40 \mathrm{~V}$ and $-80 \mathrm{~V}$ generated similar number of total protein identifications (2300 proteins). Total identified proteins and peptides decreased markedly at CVs higher than $-40 \mathrm{~V}$ and lower than $-90 \mathrm{~V}$ (Figure 2A). Interestingly, the profile of TMT-labeled peptides was generally similar to that of unlabeled peptides, though we identified labeled peptides at $-30 \mathrm{~V}$, while unlabeled peptides were not identified until $-40 \mathrm{~V}$ (Figure 2A, S1A). The intersection of peptides across CVs reached a maximum of $35 \%(-50 \mathrm{~V}$ and $-60 \mathrm{~V}$ ) but generally remained below $20 \%$ for CVs from -30 to -100 (Figure 2B). The low overlap of identifications suggested that FAIMS could separate precursors in a manner analogous to condensed phase fractionation.

The TKO standard consists of three knockout yeast strains ( $\Delta$ met6, $\Delta u r a 2, \Delta h i s 4)$ combined in triplicate with two wild-type yeast replicates (Figure S1B). Reporter ion interference can be measured using quantified TKO peptides matching to one of the knocked out proteins as the reporter ion channels for these KO strains should have no TMT reporter signal. When TMT signal is observed for these peptides in the knockout channels the signal must intrinsically be due to reporter interference, which is measured using the interference free index (IFI; IFI = 1 means no observed interference). Using the TKO standard, we observed a direct relationship between the FAIMS CV and the quantitative interference measured for individual peptides (Figure S1C). 
TKO standard IFI's for low isolation width (0.5-0.7 Th) HRMS ${ }^{2}$ methods without FAIMS have generally been observed between 0.65 and 0.8 , with variation primarily due to the chromatographic column used and/or the current performance of the instrument (Figure S2). ${ }^{14,20}$ For FAIMS-HRMS ${ }^{2}$ with a single CV, we observed the mean IFI to be similar to HRMS $^{2}$ methods without FAIMS (mean IFI $=0.720 \pm 0.025$ SEM, Figure 3A). Interestingly FAIMS-SPS-MS ${ }^{3}$ methods at single CVs consistently resulted in high IFIs for all measured CVs (mean $=0.914 \pm 0.018$ SEM, Figure 3B). FAIMS LC-MS/MS methods can alternatively cycle through multiple CVs to analyze a single sample injection. For multi-CV methods, we selected CVs with a low inter-CV identified peptide intersections and high rates of protein identifications (Figure 2A-B). With FAIMS-HRMS ${ }^{2}$ methods utilizing 2 $\mathrm{CVs}$ we observed a modest $12 \%$ increase in the IFI for TKO knockout proteins (mean = $0.726 \pm 0.008$ SEM, Figure 3C) compared with single CV methods (mean $=0.650 \pm 0.020$ SEM, Figure 3C). Strikingly, increasing the number ofCVs to 3 improved the IFI even further to $0.919 \pm 0.015$ SEM (Figure 3C). This corresponds to a $41 \%$ increase in IFI compared to single $\mathrm{CV}$ methods, and translates into a 4.3-fold reduction in TMT reporter ion interference when using three CVs with FAIMS-HRMS ${ }^{2}$. Increasing the number of CVs (i.e., $4 \mathrm{CVs}$ ) maintained the improved quantitative accuracy, however adding a fourth $\mathrm{CV}$ led to reduced total protein identifications, suggesting that $3 \mathrm{CV}$ s provides an optimal balance for quantitation and identification (Figure S2B,C).

With respect to quantitative dynamic range, for a single CV HRMS ${ }^{2}$ method the maximum observable ratio (MOR, Figure S3A, Methods) between any two reporter channels in the sample was a 2.86-fold change (MOR calculated based on the mean of single CV HRMS ${ }^{2}$ IFIs, Figure 3C). With 2CVs, the MOR was 3.66 (based on the mean IFI for $2 \mathrm{CV}$ methods, Figure $3 \mathrm{C}$ ), and for $3 \mathrm{CVs}$ the MOR was 12.35 . Thus, utilizing multiple CVs increased the quantitative dynamic range 4-fold. One factor contributing to the improvement of $3 \mathrm{CV}$ methods over 1 or $2 \mathrm{CV}$ methods was the increased median precursor intensity for the $3 \mathrm{CV}$ method compared to the 1 and $2 \mathrm{CV}$ methods. We observed a similar effect when comparing canonical SPS-MS ${ }^{3}$ methods without FAIMS, whereby peptides with high precursor purity (greater than 0.7) had higher precursor intensity (Figure S3B). Precursor purity denotes the proportion of the isolation window intensity due to the selected precursor. Rationally this can be attributed to coisolation of interfering peaks having a larger effect on low abundance precursors. Sampling precursors at three CVs enriched high abundance precursors by selecting only the "best" precursors at each mobility for quantitation. In other words, targeting the top $n$ most intense precursors at each CV limited data dependent targeting of low intensity precursors for quantitation at any individual CV.

We next examined the effect of factors that have previously been cited as contributing to precursor coisolation and quantitative interference. ${ }^{1,3,8}$ First we studied the effect of sample loading amount on FAIMS method performance. We injected varying amounts of TKO standard ( $0.9 \mathrm{ng}$ to $273 \mathrm{ng}$ per proteome in an 11-plex) using a FAIMS-HRMS ${ }^{2}$ method (Figure S4, Figure S5). FAIMS increased or matched total protein identifications for input amounts down to $2.7 \mathrm{ng}$ per proteome (30 $\mathrm{ng}$ total injection) compared with $\mathrm{HRMS}^{2}$ methods without FAIMS (Figure S4). In the range of typical large-scale TMT analyses (45 ng per proteome to $273 \mathrm{ng}$ per proteome), ${ }^{16}$ increasing the injection amount increased total peptide identifications slightly but had little effect on the observed interference or 
quantitative accuracy (Figure S5). Consistent identification rates across injection amounts demonstrates that the new FAIMS source can achieve sensitive detection of multiplexed samples down to $9 \mathrm{ng}$ per proteome, in part due to improved transmission of ions through the device. ${ }^{10-12}$

Second, we tested the effect of changing the precursor isolation widths from $0.5 \mathrm{~m} / \mathrm{z}$ to 2.0 $\mathrm{m} / \mathrm{z}$ on FAIMS methods (Figure 4A). Quantitative accuracy for FAIMS-HRMS ${ }^{2}$ methods decreased as the isolation width increased when the analysis was performed with or without FAIMS (Figure 4A). However, we observed that FAIMS-HRMS ${ }^{2}$ provided higher quantitative accuracy across all isolation widths tested. Peptides quantified using FAIMSHRMS $^{2}$ at $0.5 \mathrm{~m} / \mathrm{z}$ isolation width had increased quantitative accuracy, individual channel signal-to-noise and summed channel signal-to-noise (Figure 4B). Differential ion mobility separation of coeluting precursors improved multiplexed quantitative dynamic range and enriched TMT containing precursors within each CV. FAIMS also improved the quantitative accuracy of FAIMS-SPS-MS ${ }^{3}$ methods for all tested isolation windows (Figure 4C). Along with the improved accuracy we observed a $6 \%$ increase in total protein identifications for the $0.5 \mathrm{~m} / \mathrm{z}$ isolation width with the FAIMS-SPS-MS ${ }^{3}$ method compared to the SPS-MS ${ }^{3}$ method (Figure 4D, Figure S6).

To test if the benefits of FAIMS extended to more complex samples, we used the twoproteome HYPER standard (human/yeast TMT peptide standard). ${ }^{21,22}$ This standard provides a near real world context for quantitative accuracy assessment due to the high sample complexity and the assumption that biological perturbations only alter a subset of the analyzed proteome, here modeled as $10 \%$ of the total sampled proteome changing (yeast peptides, Figure 5A). The yeast peptides were mixed at several ratios (1, 1.5 and 3 fold changes) with no yeast peptides in the first and last channels (TMT-126 and TMT-131c, Figure 5A). The human peptides were mixed at a ratio of 1:1 across all 11 channels such that the mass of yeast peptides was $10 \%$ of the total peptide mass. Across all runs, we observed an average of $10.1 \%$ of the total peptides were identified as yeast peptides (Table S1).

For both HRMS ${ }^{2}$ and SPS-MS ${ }^{3}$ methods, incrementing the number of CVs in the method increased quantitative accuracy (Figure 5B, Table S1). For the 3-to-1 ratio yeast channels, replicate injections of the same sample at $3 \mathrm{CV}_{\mathrm{s}}$ with FAIMS-HRMS ${ }^{2}$ or FAIMS-SPS-MS ${ }^{3}$ resulted in a median observed ratio of 2.64 or 3.18, respectively. The FAIMS-SPS-MS ${ }^{3}$ method resulted in the least reporter ion interference indicated by the signal-to-noise in the "empty" TMT-126 channel (Figure 5, Figure S7). We measured this interference using an analogue to the TKO IFI, wherein the two reporter ion channels lacking yeast peptides (TMT-126 and TMT-131c) were used in place of the TKO knockout strains in the original IFI equation(Figure 5C, Figure S1B). The least interference and highest quantitative accuracy was observed for the FAIMS-SPS-MS ${ }^{3}$ methods at either $2 \mathrm{CV}$ s or $3 \mathrm{CV}$ s $(0.94$ or 0.96 , respectively, Figure 5B,C). Based on the HYPER interference free index, we calculated the MOR to be 5.3 fold for the 3CV FAIMS-HRMS ${ }^{2}$ method (IFI $=0.81$ ) and 25 fold for the FAIMS-SPS-MS ${ }^{3}$ method (IFI $=0.96$, Figure 5C). While the difference in IFI values for the FAIMS-SPS-MS ${ }^{3} 2 \mathrm{CV}(0.94)$ and $3 \mathrm{CV}(0.96)$ methods was small, this represented a change in the MOR from 17 to 25, respectively (Figure S3A). As noted above, 
the improved quantitative accuracy with increasing numbers of $\mathrm{CV}$ was in part due to higher precursor intensities and precursor isolation purity (Figure S7D, Figure S8).

\section{CONCLUSIONS}

After optimizing our methods for sensitivity and quantitative accuracy, we recommend that FAIMS methods using HRMS $^{2}$-only quantification should use at least $3 \mathrm{CV}$ and maintain an isolation window as small as possible based on the instrument, with $0.5 \mathrm{~m} / \mathrm{z}$ or below being ideal to ensure the greatest gains in quantitative accuracy. For FAIMS-SPS-MS ${ }^{3}$ methods the use of at least $2 \mathrm{CVs}$ improved peptide and protein identification rates and simultaneously improved quantitative accuracy. Further we suggest using $\mathrm{CV}$ values between -40 and $-80 \mathrm{CV}$ for maximal protein identifications with TMT-labeled peptides, though using a CV of -90 may be helpful for some peptides.

For multiplexed quantitative proteomics, FAIMS $\left(\mathrm{HRMS}^{2}\right.$ or SPS-MS $\left.{ }^{3}\right)$ improved quantitative accuracy without sacrificing protein identifications. FAIMS-SPS-MS ${ }^{3}$ consistently had the least quantitative interference, followed by (in order) non-FAIMS-SPS$\mathrm{MS}^{3}$, FAIMS-HRMS ${ }^{2}$, and non-FAIMS-HRMS ${ }^{2}$ (Figure 4A-C, 5B,C). Significant reduction in reporter ion interference, as observed with FAIMS methods, has the potential to enable experiments that were previously unattractive for multiplexed quantification, including samples with high abundance peptides that elute across broad sections of the chromatogram (e.g., plasma or unfractionated lysates).

\section{Supplementary Material}

Refer to Web version on PubMed Central for supplementary material.

\section{ACKNOWLEDGMENTS}

We would like to thank members of the Gygi lab for helpful discussion, particularly Drs. Ed Huttlin, David Nusinow, and Julien Mintseris for additional technical assistance. Funding sources include K01 DK098285 (J.A.P.) and U24 HG006673 07 (S.P.G.).

\section{REFERENCES}

(1). Hogrebe A; von Stechow L; Bekker-Jensen DB; Weinert BT; Kelstrup CD; Olsen JV Nat. Commun 2018, 9, 1045. [PubMed: 29535314]

(2). Rauniyar N; Yates JR 3rd. J. Proteome Res 2014, 13, 5293-5309. [PubMed: 25337643]

(3). Ting L; Rad R; Gygi SP; Haas W Nat. Methods 2011, 8, 937-940. [PubMed: 21963607]

(4). Pfammatter S; Bonneil E; Thibault P J. Proteome Res 2016, 15, 4653-4665. [PubMed: 27723353]

(5). Savitski MM; Mathieson T; Zinn N; Sweetman G; Doce C; Becher I; Pachl F; Kuster B; Bantscheff M J. Proteome Res 2013, 12, 3586-3598. [PubMed: 23768245]

(6). Wenger CD; Lee MV; Hebert AS; McAlister GC; Phanstiel DH; Westphall MS; Coon JJ Nat. Methods 2011, 8, 933-935. [PubMed: 21963608]

(7). Wuhr M; Haas W; McAlister GC; Peshkin L; Rad R; Kirschner MW; Gygi SP Anal. Chem 2012, 84, 9214-9221. [PubMed: 23098179]

(8). McAlister GC; Nusinow DP; Jedrychowski MP; Wuhr M; Huttlin EL; Erickson BK; Rad R; Haas W; Gygi SP Anal. Chem 2014, 86, 7150-7158. [PubMed: 24927332]

(9). Pfammatter S; Bonneil E; McManus FP; Prasad S; Bailey DJ; Belford M; Dunyach JJ; Thibault P Mol. Cell. Proteomics 2018, 17, 2051. [PubMed: 30007914] 
(10). Hebert AS; Prasad S; Belford MW; Bailey DJ; McAlister GC; Abbatiello SE; Huguet R; Wouters ER; Dunyach JJ; Brademan DR; Westphall MS; Coon JJ Anal. Chem 2018, 90, 9529-9537. [PubMed: 29969236]

(11). Prasad S; Belford MW; Dunyach JJ; Purves RW J. Am. Soc. Mass Spectrom 2014, 25, $2143-$ 2153. [PubMed: 25267086]

(12). Purves RW; Prasad S; Belford M; Vandenberg A; Dunyach JJ J. Am. Soc. Mass Spectrom 2017 , 28, 525-538. [PubMed: 28097537]

(13). Pfammatter S; Bonneil E; McManus FP; Prasad S; Bailey DJ; Belford M; Dunyach JJ; Thibault P Mol. Cell. Proteomics 2018, 17, 2051-2067. [PubMed: 30007914]

(14). Paulo JA;O’Connell JD; Gygi SP J. Am. Soc. Mass Spectrom 2016, 27, 1620-1625. [PubMed: 27400695]

(15). Eng JK; McCormack AL; Yates JR J. Am. Soc. Mass Spectrom 1994, 5, 976-989. [PubMed: 24226387]

(16). Navarrete-Perea J; Yu Q; Gygi SP; Paulo JA J. Proteome Res 2018, 17, 2226-2236. [PubMed: 29734811]

(17). Elias JE; Gygi SP Nat. Methods 2007, 4, 207-214. [PubMed: 17327847]

(18). Huttlin EL; Jedrychowski MP; Elias JE; Goswami T; Rad R; Beausoleil SA; Villen J; Haas W; Sowa ME; Gygi SP Cell 2010, 143, 1174-1189. [PubMed: 21183079]

(19). R Core Team. R: A Language and Environment for Statistical Computing; R Foundation for Statistical Computing: Vienna, Austria, 2018.

(20). Myers SA; Klaeger S; Satpathy S; Viner R; Choi J; Rogers J; Clauser K; Udeshi ND; Carr SA J. Proteome Res 2018, DOI: 10.1021/acs.jproteome.8b00611.

(21). O'Brien JJ; O'Connell JD; Paulo JA; Thakurta S; Rose CM; Weekes MP; Huttlin EL; Gygi SP J. Proteome Res 2018, 17, 590-599. [PubMed: 29195270]

(22). O’Connell JD; Paulo JA; O’Brien JJ; Gygi SP J. Proteome Res 2018, 17, 1934-1942. [PubMed: 29635916] 

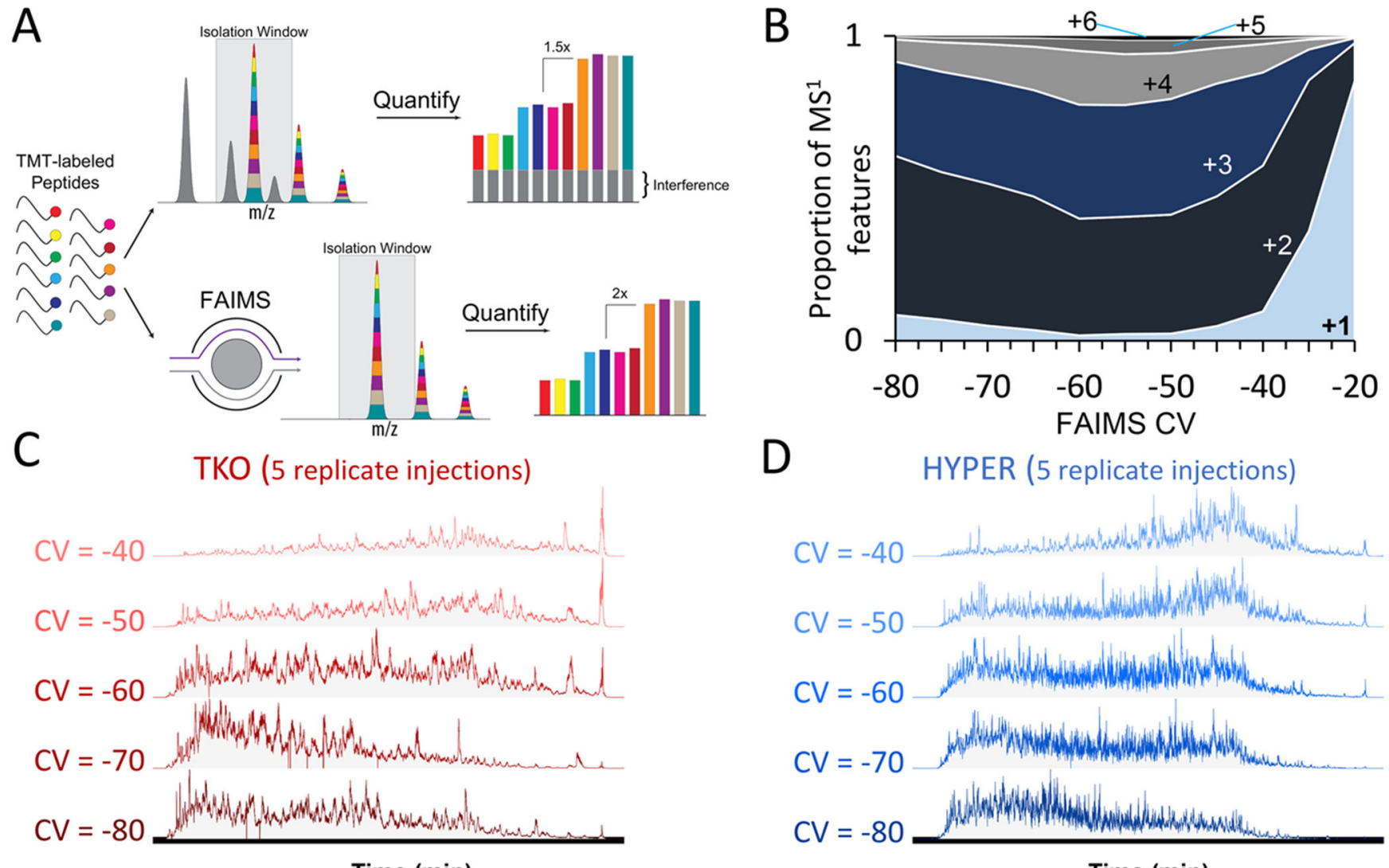

$\mathrm{D}$

HYPER (5 replicate injections)

Time (min)

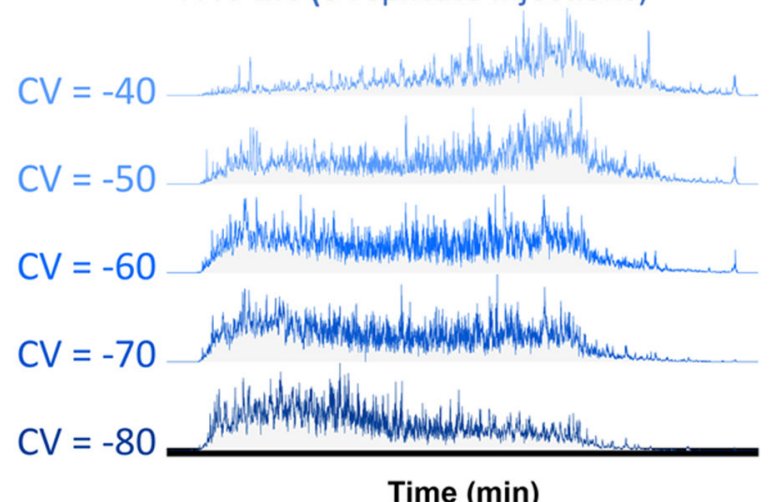

Figure 1.

Mobility-based separation of precursors. (A) Peptides are selectively filtered based on the compensation voltages (CVs) applied to the FAIMS source. In the upper panel, interference due to the gray coisolated ions cause suppression of the ratio measured as a 1.5-fold change. FAIMS eliminates interference due to precursor coisolation resulting in a true ratio, in this case a 2-fold change. (B) Proportion of $\mathrm{MS}^{1}$ features at each CV based on their charge. HYPER standard precursor centroids fractionate partially by charge over different CVs. Charge +1 features were destabilized at CVs less than $-30 \mathrm{~V}$. Charge +2 features were enriched at $\mathrm{CVs}$ less than $-30 \mathrm{~V}$. Charge +3 features were enriched at CVs less than $-40 \mathrm{~V}$. (C) and (D) Chromatograms for TKO and HYPER standard replicate injections across multiple $\mathrm{CV}$ values. 

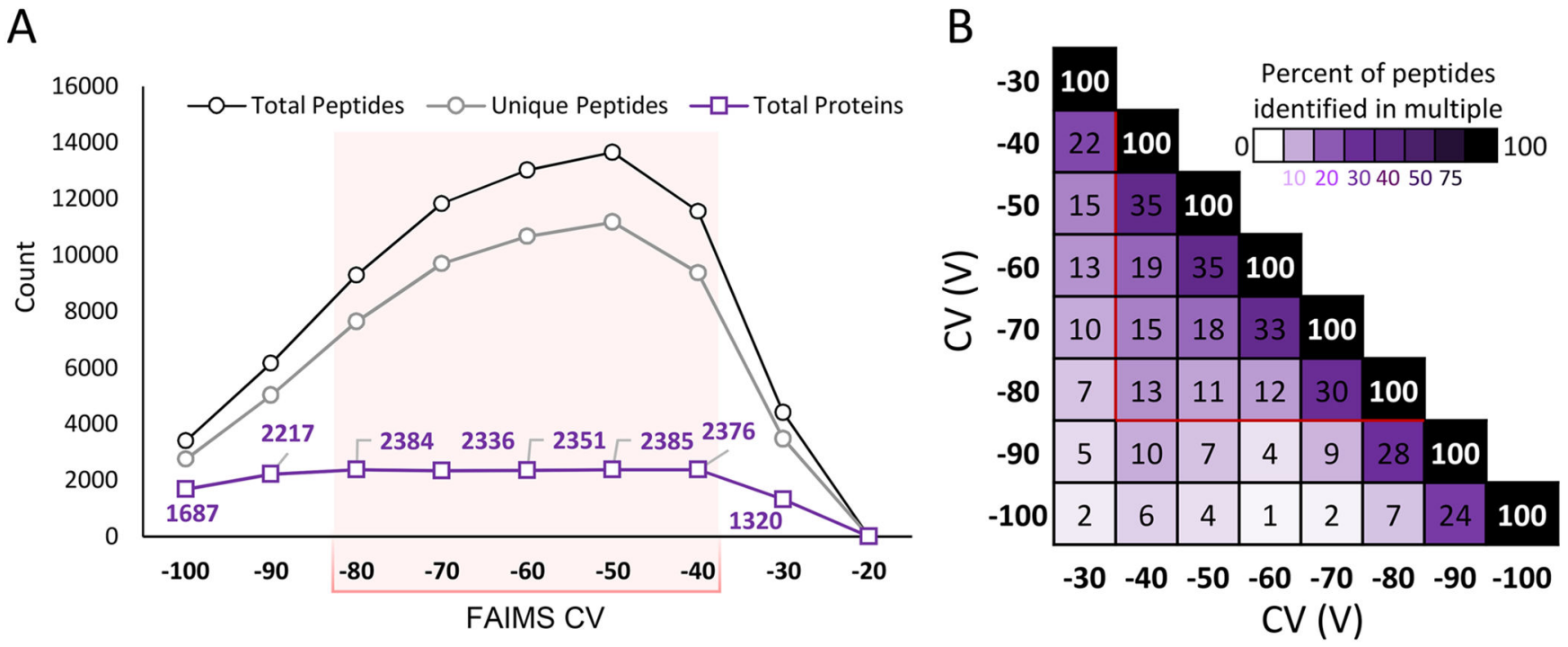

Figure 2.

Peptide and protein identifications across FAIMS CVs. (A) Peptide, unique peptide, and protein identification rates for each tested CV from -20 to -100 . Protein identification rates were stable from -40 to $-80 \mathrm{~V}$. (B) Peptide level overlap between replicate injections of TKO across CVs. For replicate injections of the same sample, the maximum peptide overlap between single CV methods was $35 \%$. 

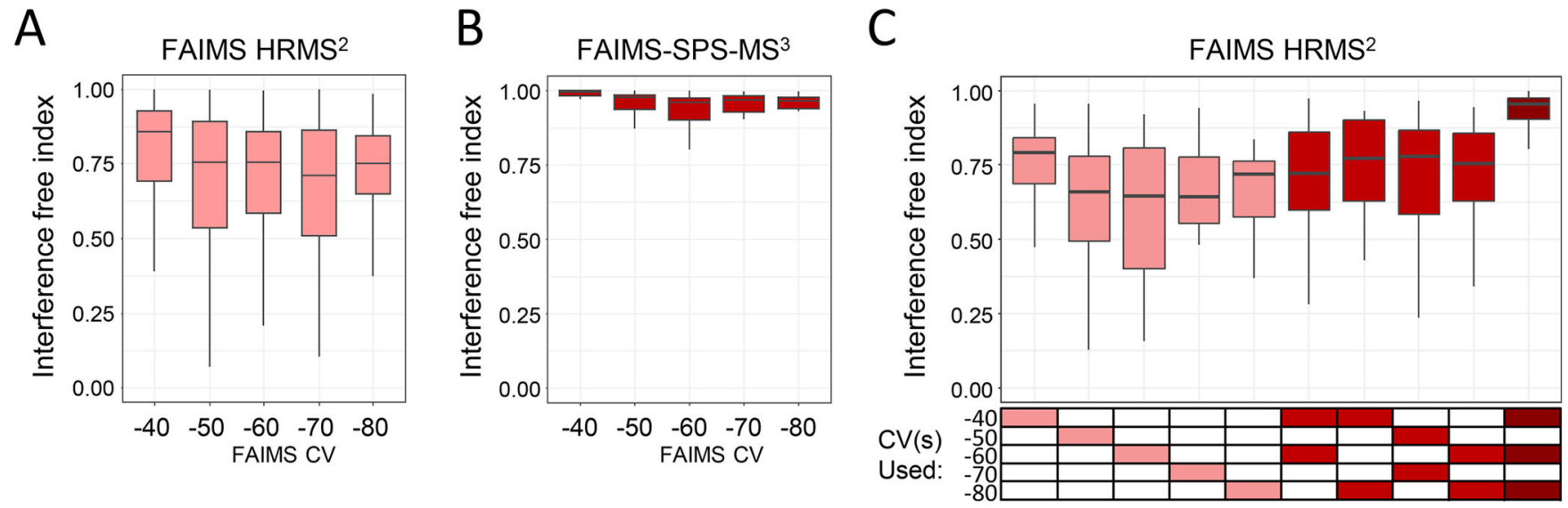

Figure 3.

Comparison of reporter ion interference observed for FAIMS methods. Median $\Delta$ met6 channel IFI values in red are shown for each comparison. (A) Interference for HRMS ${ }^{2}$ methods across multiple $\mathrm{CV}$ values using only one $\mathrm{CV}$ at a time. (B) Interference for SPS$\mathrm{MS}^{3}$ methods across multiple $\mathrm{CV}$ values using only one $\mathrm{CV}$ at a time. SPS-MS ${ }^{3}$ improved IFI values compared to $\mathrm{HRMS}^{2}$, consistent with previous reports. (C) Measured IFI for FAIMS-HRMS ${ }^{2}$ methods applying cycles of one (pink), two (red) or three CVs (dark red). $\mathrm{CV}$ s for each method are depicted in the table below. 
A

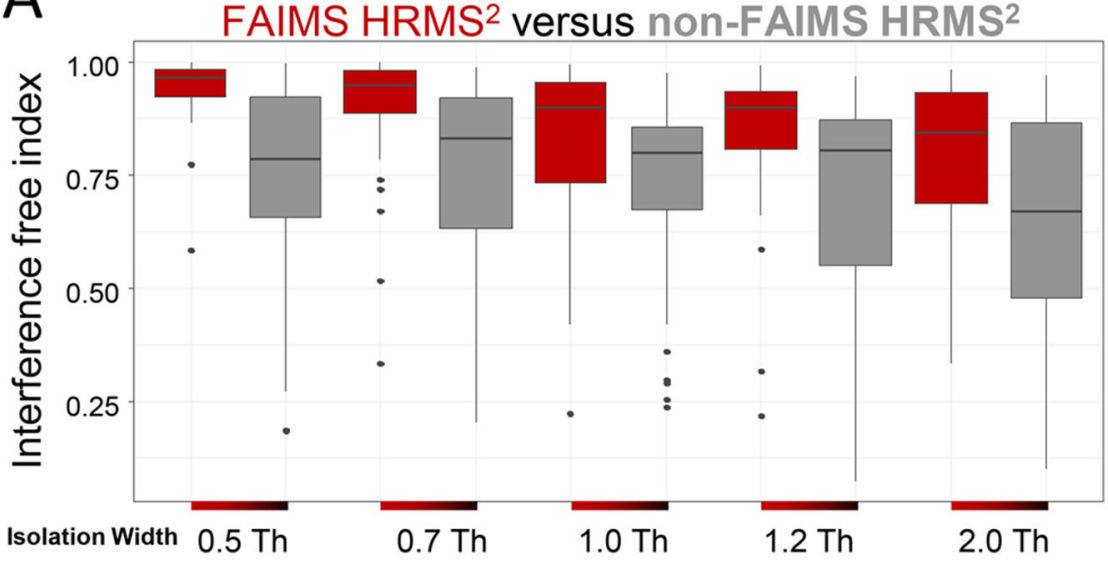

C

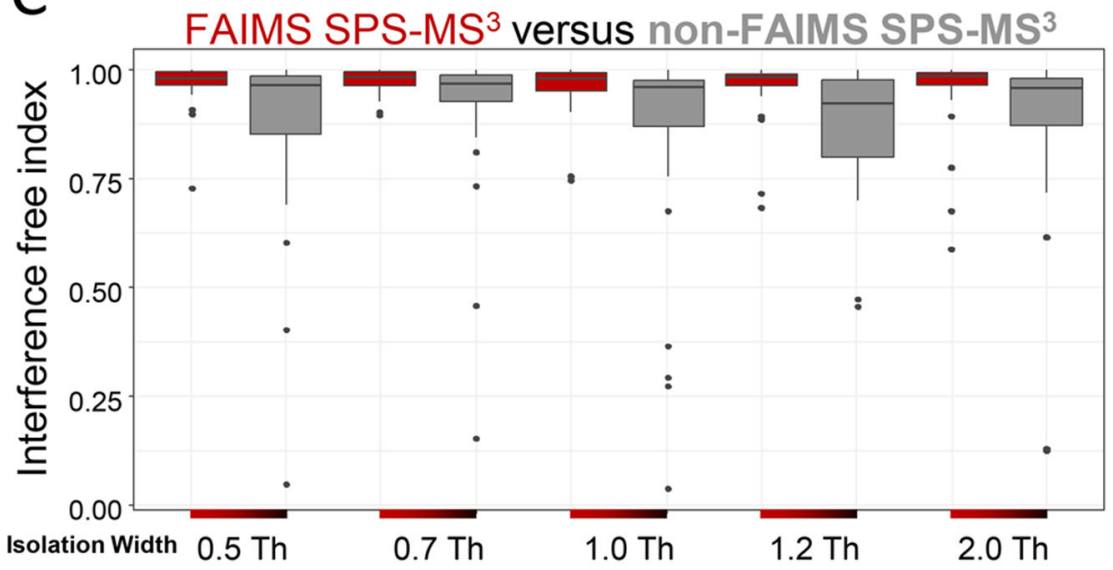

Figure 4.
B
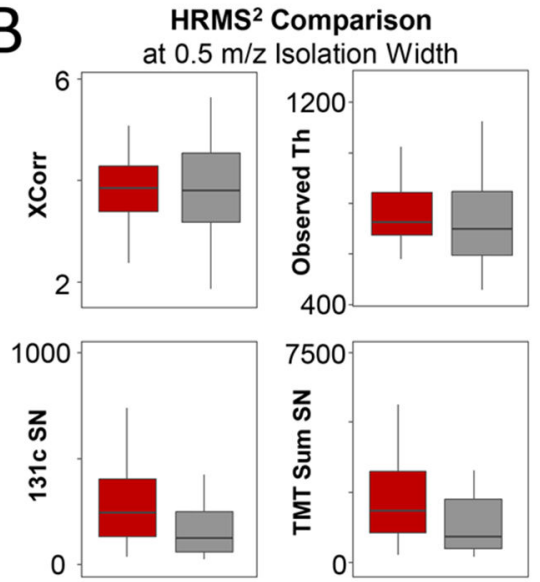

$\mathrm{D}$

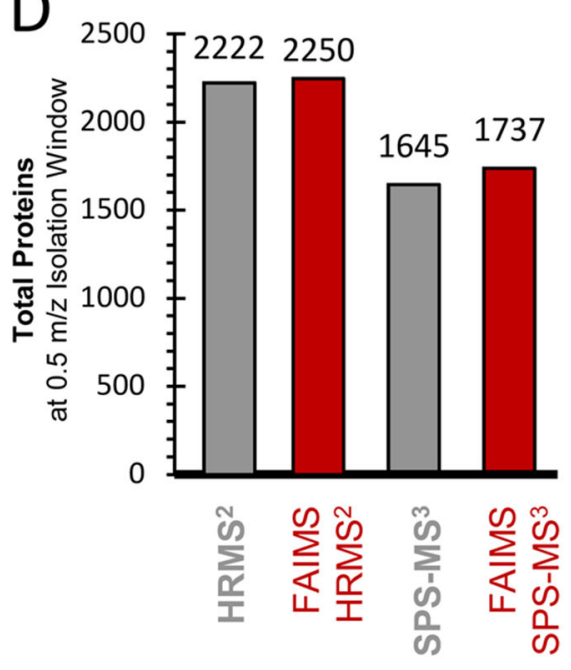

Optimization of HRMS ${ }^{2}$ and SPS-MS ${ }^{3}$ methods for multiplexed quantification. (A) IFI measurements for the $\Delta$ met6 strain channels across multiple isolation widths for HRMS ${ }^{2}$ based methods. FAIMS data are shown as red boxes. Non-FAIMS data are shown as gray boxes. (B) Comparison of multiple metrics for the $0.5 \mathrm{~m} / z$ FAIMS-HRMS ${ }^{2}$ (red) versus HRMS $^{2}$ (gray). (C) IFI measurements for the $\Delta$ met6 $\mathrm{KO}$ strain across multiple isolation widths for SPS-MS ${ }^{3}$-based methods. FAIMS data are shown as red boxes. Non-FAIMS data are shown as gray boxes. Values are median IFI for the given experiment. (D) Total identified proteins for the HRMS $^{2}$, SPS-MS ${ }^{3}$, FAIMS-HRMS ${ }^{2}$ and FAIMS-SPS-MS ${ }^{3}$ methods using a $0.5 \mathrm{~m} / \mathrm{z}$ isolation width. 
A
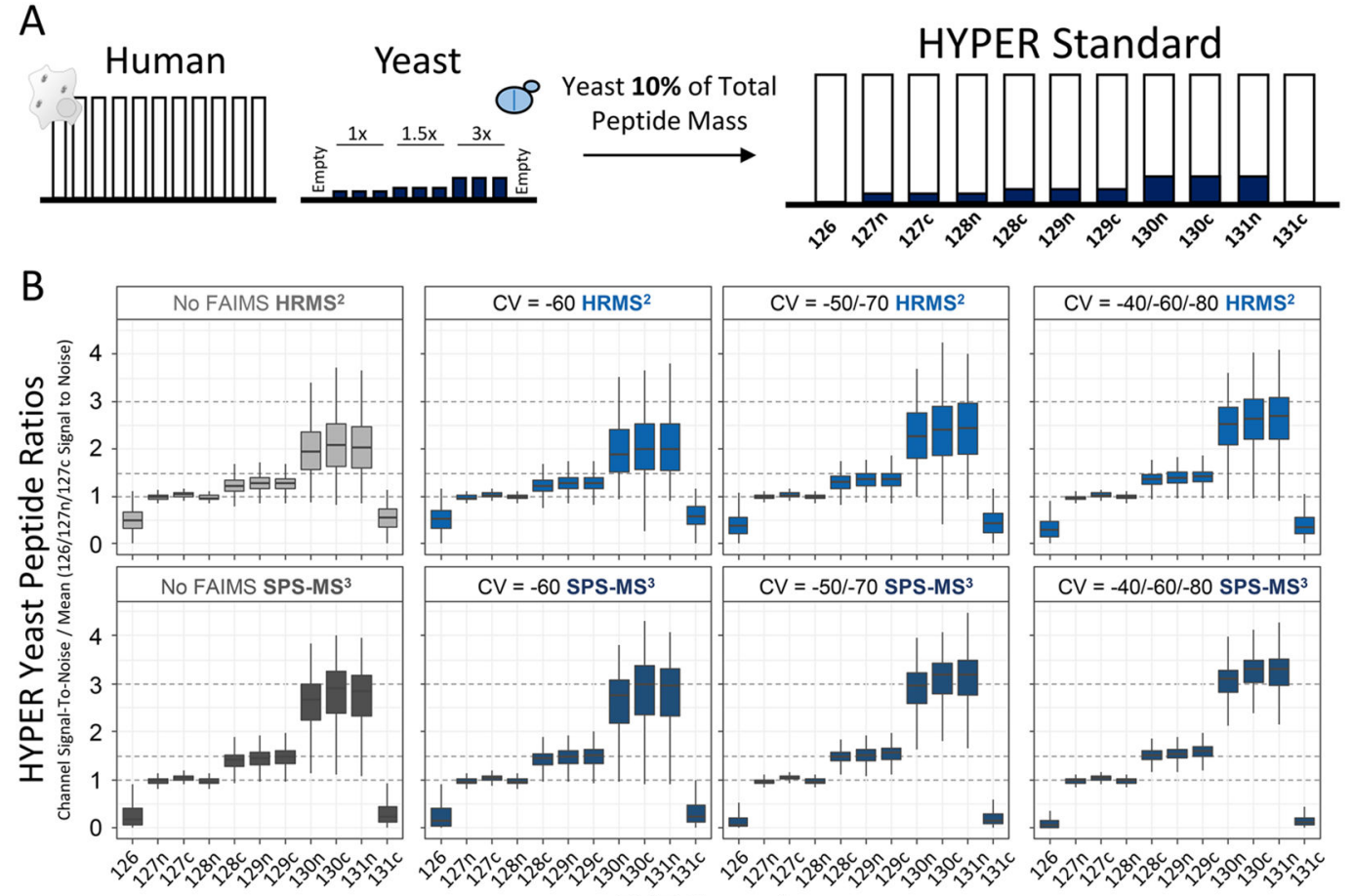

TMT Channels

C

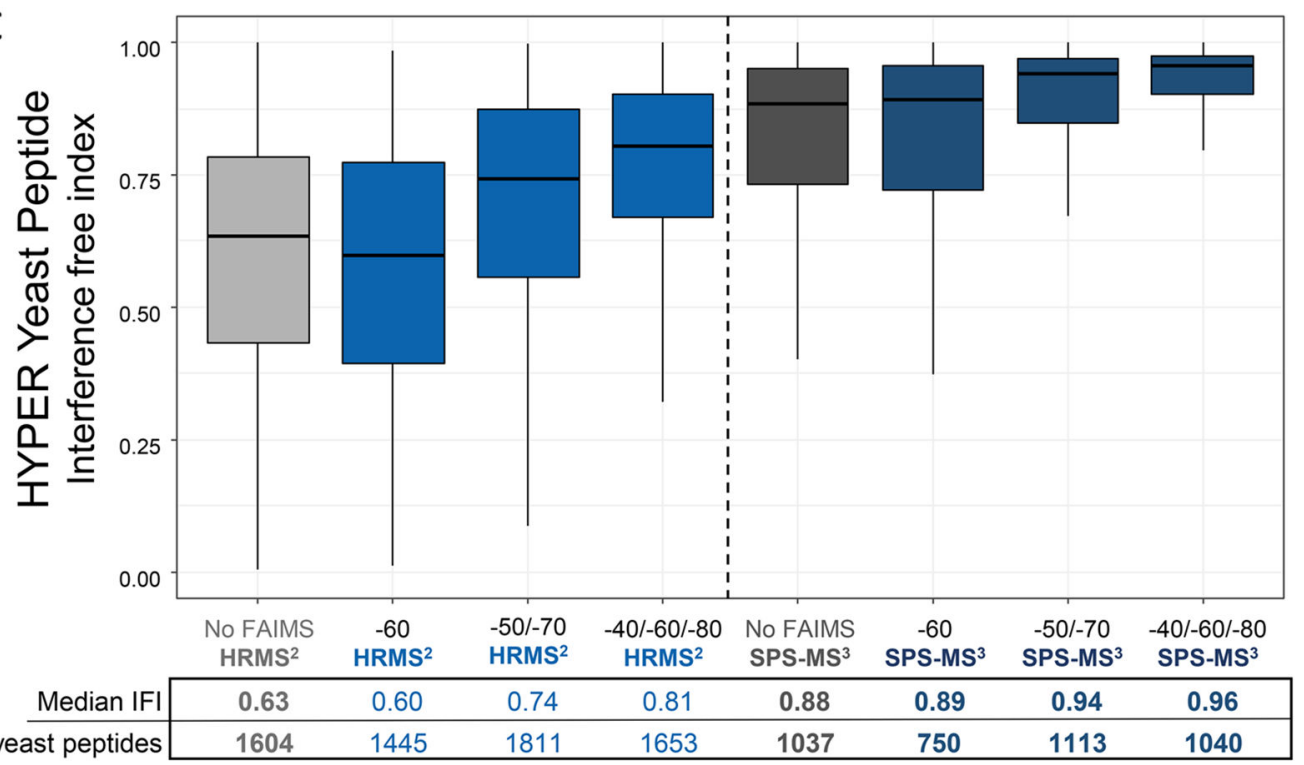

Figure 5.

Quantification of complex biological samples is improved using multiple FAIMS CVs. (A) The HYPER standard is a novel means to determine small numbers of changing protein abundances in background. $1.1 \mathrm{mg}$ of human protein was equally divided at a 1:1 ratio across 11 TMT channels. 110ug of yeast peptides were mixed into the human background at varying concentrations to represent $\sim 10 \%$ of a proteomic sample changing. (B) Quantified yeast peptide ratios across multiple HRMS $^{2}$ and SPS-MS ${ }^{3}$ methods. Expected ratios (1, 1.5 or 3) for the three triplicate yeast peptide concentrations are marked with dotted gray lines. 
Using multiple CVs improved both HRMS ${ }^{2}$ and SPS-MS ${ }^{3}$ analyses. (C) Interference free index (IFI) calculated using the empty HYPER standard channels (TMT-126 and TMT-131c) as the "knockout" channels in the canonical calculation. Median IFI, total number of yeast peptides (n peptides) and CVs used are shown. 